\section{A interpretação das novas diretrizes para a detecção precoce do câncer de mama no Brasil}

Interpretation of the new guidelines for breast cancer early detection in Brazil

La interpretación de las nuevas directrices para la detección temprana del cáncer de mama en Brasil

Arn Migowski 1,2

${ }_{1}^{1}$ Instituto Nacional de Câncer José Alencar Gomes da Silva, Rio de Janeiro, Brasil.

2 Instituto Nacional de Cardiologia, Rio de Janeiro,

Brasil.

\section{Correspondência}

A. Migowski

Divisão de Detecção Precoce e Apoio à Organização de Rede, Instituto Nacional de Câncer José Alencar Gomes da Silva.

Rua Marquês de Pombal 125, Rio de Janeiro, RJ

20230-240, Brasil.

arnmigowski@yahoo.com.br

http://dx.doi.org/10.1590/0102-311X00111516

O ensaio de Tesser \& d'Ávila traz uma discussão muito bem-vinda, relevante e relativamente pouco abordada na literatura nacional 1 . Contudo, há um equívoco no mote do artigo, uma vez que as diretrizes nacionais de detecção precoce do câncer de mama acabam de ser revistas, contemplando todas as questões colocadas pelos autores e chegando a conclusões muito próximas, ao contrário da interpretação feita por eles 2 .

Com relação à eficácia do rastreamento, o ensaio cita vários estudos observacionais e resultados isolados de ensaios clínicos, ao mesmo tempo que contraditoriamente defende que " $a$ decisão deve ser estritamente coerente com e dependente do melhor, mais sólido e atualizado saber científico disponível" 1 (p. 3) e baseada no resultado de "metanálises de ensaios clínicos". As novas diretrizes nacionais foram baseadas na revisão mais extensa já feita no Brasil sobre riscos e benefícios da detecção precoce do câncer de mama, e os resultados de eficácia do rastreamento foram baseados em revisões sistemáticas de ensaios clínicos.

Avaliando a metanálise dos ensaios clínicos randomizados de rastreamento mamográfico, são encontrados estudos com resultados homogêneos e uma medida-sumário de efeito estatisticamente significativa ${ }^{3}$. Essa consistência fala a favor da existência real de efeito na redução da mortalidade por câncer de mama, a despeito da existência de variação de qualidade entre os ensaios clínicos. Na verdade, a citada crítica da revisão Cochrane no que concerne à qualidade de alguns ensaios clínicos não é nova, mas vem desde a revisão de 2001. Mesmo assim, a versão atual da revisão Cochrane ainda reconhece a evidência de eficácia do rastreamento ainda que de peque- na magnitude ${ }^{3}$. Nas novas diretrizes nacionais, essa consistência foi valorizada e não as evidências "consensuais" defendidas no ensaio, pois não se tratou de consenso de opinião de especialistas 1 .

Na avaliação do equilíbrio entre danos e benefícios, as novas diretrizes incluíram o efeito absoluto do rastreamento na mortalidade e todos os danos citados pelos autores do ensaio, sendo ainda mais abrangente por ter incluído outros riscos, como a possibilidade de indução de câncer pela radiação ionizante dos exames de mamografia. As recomendações das novas diretrizes seguiram a mesma lógica utilizada pelos autores do ensaio. No caso de balanço desfavorável entre danos e benefícios, o rastreamento foi contraindicado, exatamente como ocorreu na recomendação para mulheres com menos de 50 anos ${ }^{2}$. No caso de "equilíbrio" entre danos e benefícios ou fraca vantagem dos benefícios, a recomendação foi "traduzida como indicando decisão compartilhada e singularizada entre usuário e profissional". Essa é exatamente uma das implicações da recomendação favorável fraca adotada para o rastreamento de 50-69 anos nas novas diretrizes. Tais recomendações têm ainda diversas outras implicações para gestores, profissionais de saúde e população que não poderão ser esgotadas no presente texto.

Tesser \& d'Ávila 1 afirmam ainda que estudos mostraram não haver redução da mortalidade geral com o rastreamento, quando o que há de fato é ausência de significância estatística a respeito de um desfecho insuficientemente estudado. Na ausência desse desfecho ideal, muitas tentativas de determinar o balanço entre danos e benefícios do rastreamento são flagrantemente frágeis como o critério citado pela Organização Mundial da Saúde de que dois casos de sobrediagnóstico para cada morte por câncer de mama evitada seriam aceitáveis 1 . Todavia, da mesma forma é questionável arbitrar que dez casos de sobrediagnóstico por uma vida prolongada representam um balanço negativo como parecem sugerir os autores. Aqui se chega ao cerne do debate: esses desfechos não são diretamente comparáveis entre si e esse é um limite claro das evidências disponíveis.

A estratégia de agilização da confirmação diagnóstica, apontada como prioritária por Gebrim 4 , foi incluída nas novas diretrizes nacionais de forma pioneira e só não ganhou mais espaço pela debilidade das evidências nacionais e internacionais sobre sua eficácia. Infelizmente, os resultados de distribuição de estádios apresentados por Gebrim 4 têm pouco significado na avaliação da efetividade da intervenção proposta, pois a coexistência de rastreamento certamente inflou a proporção de casos em estádio inicial. Além disso, é preciso ter cuidado na implementação dessa estratégia porque, num contexto de grande disseminação do rastreamento, corre-se o risco de perpetuar e de até mesmo aumentar iniquidades se a urgência na investigação não for direcionada às mulheres com sinais e sintomas suspeitos. Isso tende a ocorrer naturalmente, já que os casos positivos no rastreamento são muito numerosos, e essas mulheres já estão inseridas no sistema de saúde e por isso com mais facilidade de acesso do que as mulheres sintomáticas, justamente as que mais se beneficiariam. 
Ao contrário do que afirmou Gebrim ${ }^{4}$, não foi feita validação de diretrizes internacionais pelo Ministério da Saúde brasileiro. A maior parte das diretrizes internacionais é elaborada por consenso de especialistas, sendo por conta disso muito menos válidas metodologicamente do que as novas diretrizes nacionais. Inclusive não foi validada nem mesmo a diretriz da U.S. Preventive Services Task Force - considerada por muitos a melhor já feita - conforme explicitado recentemente em um comentário para o editor do periódico Annals of Internal Medicine 5 . É preciso ainda enfatizar que nunca houve evidência suficiente para recomendar o rastreamento anual e o rastreamento antes dos 50 anos de idade. Essa prática nunca deveria ter sido introduzida no Brasil e deve ser desencorajada. Por isso, não é possível concordar com a afirmação de Gebrim de que o rastreamento anual a partir dos 40 anos "deve secundariamente ser considerado até que se conheça melhor a custolefetividade" 4 (p. 2). Essa afirmação é fruto do conceito de priorização da faixa etária de 50-69 anos usado por Gebrim \& Quadros ${ }^{6}$, que também é equivocado por pressupor que se deseja rastrear mulheres com menos de 50 anos. Antes de qualquer consideração sobre custo, deve-se assumir que o balanço entre riscos e benefícios é favorável, o que não acontece nesse caso. Portanto, indicadores como o percentual de carcinoma ductal in situ e cobertura de rastreamento em mulheres acima de 40 anos - considerados por Gebrim ${ }^{7}$ como positivos - não devem ser adotados.

O presente debate felizmente revela que há muito mais concordâncias com as novas diretrizes do que divergências, o que potencialmente abre espaço para sinergia entre diversos atores. O que há ainda é uma deficiência na interpretação das novas diretrizes mesmo por especialistas no tema. Para superar esse impasse, é necessário aprofundar ainda mais esse debate com a comunidade acadêmica nacional, publicando com mais detalhes reflexões sobre as consequências práticas das decisões metodológicas adotadas no processo de elaboração das novas diretrizes, a interpretação de todas as novas recomendações para os diversos atores envolvidos, os desafios de implementação e suas consequências para o Sistema Único de Saúde. Quem sabe assim se possa focar em estratégias prioritárias e superar a infundada controvérsia sobre faixa etária e periodicidade de rastreamento, que resultaram na divulgação massiva de informações equivocadas para mulheres e profissionais de saúde e prejudicaram mais de uma década de controle do câncer de mama no Brasil.
1. Tesser CD, d'Ávila TLC. Por que reconsiderar a indicação do rastreamento do câncer de mama? Cad Saúde Pública 2016; 32:e00095914.

2. Migowski A, Dias MBK, organizadores. Diretrizes para a detecção precoce do câncer de mama no Brasil. Rio de Janeiro: Instituto Nacional de Câncer José Alencar Gomes da Silva; 2015.

3. Gøtzsche PC, Jørgensen KJ. Screening for breast cancer with mammography. Cochrane Database Syst Rev 2011; (6):CD001877.

4. Gebrim LH. A detecção precoce do câncer de mama no Brasil. Cad Saúde Pública 2016; 32:eCO010516.

5. Migowski A, Silva GA, Eluf-Neto J. Breast cancer screening: different contexts require different guidelines. http://annals.org/article. aspx? articleid $=2480757$ (acessado em 27/ Jun/2016).

6. Gebrim LH, Quadros LGA. O rastreamento do câncer de mama no Brasil. Rev Bras Ginecol Obstet 2006; 28:319-23.

7. Gebrim LH. Rastreamento para câncer de mama e estadiamento. Rev Bras Ginecol Obstet 2009; 31:216-8.

Recebido em 27/Jun/2016

Aprovado em 01/Jul/2016 
The author reply

$O$ autor responde

El autor responde

Luiz Henrique Gebrim 1,2

1 Universidade Federal de São Paulo, São Paulo, Brasil. 2 Hospital Estadual Pérola Byington, São Paulo, Brasil.

\author{
Correspondência \\ L.H. Gebrim \\ Rua Mato Grosso 306, cjs 1611/12, São Paulo \\ SP 01239-040, Brasil \\ lgebrim@uol.com.br
}

Diretrizes para detecção precoce do câncer de mama no Brasil

Apesar do expressivo número de especialistas e do grande volume de recursos investidos em oncologia no Brasil, não há estatísticas nem plano estratégico voltado para detecção precoce para o câncer de mama. Observa-se na população assistida pelo Sistema Único de Saúde (SUS) uma elevada taxa de mortalidade semelhante aos países da África, Ásia e Oriente Médio ${ }^{1 .}$.

São poucas as publicações nacionais voltadas para a articulação de uma rede oncológica que visa prioritariamente à detecção precoce, sendo escassas as oportunidades para se debater tão relevante tema, o que muito contribui para aprimorar as políticas públicas de saúde aos gestores do SUS 2 .

Apesar das falhas metodológicas de Diretrizes obtidas em Consenso de especialistas como a Task Forces (Estados Unidos/Canadá), apontada por Migovski, tais Diretrizes, quando fundamentadas em dados estatísticos reais, auxiliam muito a tomada de decisões estratégicas aos gestores, especialmente em países como o Brasil, onde há grande disparidade regional de prioridades, recursos humanos e infraestrutura.

Isso explica em parte os insucessos de projetos governamentais pregressos que objetivavam implantar o rastreamento mamográfico no Brasil. Apesar do expressivo investimento para aquisição de mamógrafos, a inoperância ou subutilização deles pouco ou nada contribuiu para reduzir o grande número de casos avançados na última década, em especial nas regiões Norte e Nordeste 1,2 .

$\mathrm{Na}$ ausência de dados oficiais, indicadores institucionais das Unidades de Assistência de Alta Complexidade (UNACON) ou Centros de Assistência de Alta Complexidade em Oncologia (CACONS), devem ser divulgados com intuito de se avaliar regionalmente a incidência, estadiamento e fluxo dos pacientes para identificar os gargalos existentes e implantar medidas que possam rapidamente reduzir a mortalidade 3 .

Há consenso de que a primeira etapa custo-efetiva a ser ofertada aos usuários do SUS seria a redução de casos avançados, com programas de educação à população-alvo e capacitação aos profissionais da atenção primária, oferecendo acesso imediato às pacientes sintomáticas, exame clínico das mamas e resolutividade diagnóstica (mamografia, ultrassom e biópsia). A abreviação dessas etapas comprovadamente reduz a progressão tumoral e ocasiona decréscimo da mortalidade, conhecido como dowstage.

Tal estratégia proporcionou uma redução no número de casos avançados em países como Índia e China e na Ásia ${ }^{3}$. Concorda-se aqui com Migowski que tais medidas precisariam ser implementadas no Brasil antes do rastreamento mamográfico, aumentando a resolutividade diagnóstica e terapêutica, respectivamente nas média e alta complexidades. Entretanto, excluindo-se os casos de carcinoma in situ de nossa casuística, o atendimento resolutivo permitiu tratar 57,6\% das pacientes nos Estádios I e II e apenas $33,4 \%$ nos estádios III e IV, se se comparar com os dados oficiais de vários Estados brasileiros.

Discorda-se da afirmação de Migowski que as pacientes com alterações mamográficas suspeitas, por já estarem no sistema de saúde, sejam atendidas mais rapidamente do que aquelas com alterações clínicas. Se há grande demanda reprimida para um procedimento mais simples (biópsia core) para confirmação diagnóstica dos nódulos palpáveis suspeitos, a dificuldade de se conseguir uma biópsia estereotáxica pelo SUS é muito maior e não está disponível na maioria dos municípios e capitais brasileiras.

A investigação só ocorre em poucos centros de alta complexidade (CACONS ou UNACONS) ou laboratórios privados que dispõem de equipamentos e equipe treinada para realizar a mamotomia ambulatorial. Não raramente, a investigação é realizada cirurgicamente (agulhamento) por judicialização. O custo é três vezes superior ao da ambulatorial por demandarem internação hospitalar. Ressalte-se que a inserção de tais pacientes no Hospital terciário também contribui para aumentar o tempo de espera para cirurgia das pacientes com doença avançada, fato vivenciado por nós antes da implementação da biópsia vácuo-assistida em 20123.

Concorda-se com Migowski que o rastreamento oportunístico preconizado pelo SUS foi prematuro na última década e restrito a uma pequena parcela da população brasileira. A falta de um sistema integrado de informatização impede de saber seu custo-efetividade. Contudo, apesar de alguns Estados da Federação terem diferentes prioridades, discorda-se, em que pese o conceito da equidade e universalização do SUS, de que não deva ser oferecido uma vez que comprovadamente salvam vidas 4,5 .

Apesar de controverso no Canadá e Europa, o custo-benefício do rastreamento antes dos 50 anos, cerca de $41 \%$ dos casos diagnosticados e tratados em nossa instituição, foram em mulheres com idade inferior a 50 anos, sendo $29 \%$ entre 40 e 50 anos. Algumas publicações mostram que a curva de incidência de câncer de mama nas mulheres latino-americanas é quatro anos inferior à média europeia ${ }^{6}$. Esses achados revelam que, apesar do baixo custo-efetividade do rastreamento nesse grupo etário, não é equivocado desconsiderá-las na busca de alternativas de inclusão nos programas de rastreamento. Modelos alternativos a essa população vêm sendo testado no Canadá onde a mamografia realizada a cada dois anos e o exame clínico das mamas anual foi custo-efetiva na população entre 40 e 69 anos, mesmo com uma adesão de $61 \% 5$. 
Prematuro julgar o carcinoma in situ como overdiagnosis, em especial nas mulheres com idade entre 40 e 50 anos, por se tratar de heterogêneo grupo histopatológico em que alguns recidivam mesmo após o tratamento clássico, com mortalidade de $3 \% 8$.

1. Khokher S, Qureshi MU, Mahmood S, Sadiq S. Determinants of advanced stage at initials diagnosis of breast cancer in Pakistan; adverse tumor biology vs delay in diagnosis. Asian Pac J Cancer Prev 2016; 17:759-65.

2. Tesser CD, d'Ávila TLC. Por que reconsiderar a indicação do rastreamento do câncer de mama? Cad Saúde Pública 2016; 32:e00095914.

3. Gebrim LH, Shida JY, Hegg R, Topis T, Mattar A. Avaliação do tempo de início do tratamento, estadiamento histopatológico e positividade dos biomarcadores (RE, RP, HER-2) em 3.566 pacientes tratadas pelo SUS no período de 2012 a 2014, no Hospital Pérola Byington. Rev Bras Mastologia 2014; 24:65-9.

4. Pataky R, Phillips N, Peacock S, Coldman AJ. Costeffectiveness of population-based mammography screening strategies by age range and frequency. J Cancer Policy 2014; 2:97-102.

5. Mittmann N, Stout NK, Lee P, Tosteson AN, Trentham-Dietz A, Alagoz O, et al. Total cost-effectiveness of mammography screening strategies. Health Rep 2015; 26:16-25.

6. Franco-Marina F, López-Carrillo L, Keating NL, Arreola-Ornelas H, Marie Knaul F. Breast cancer age at diagnosis patterns in four Latin American Populations: a comparison with North American countries. Cancer Epidemiol 2015; 39:831-7

7. Sanders ME, Schuyler PA, Simpson JF, Page DL, Dupont WD. Continued observation of the natural history of low-grade ductal carcinoma in situ reaffirms proclivity for local recurrence even after more than 30 years of follow-up. Mod Pathol 2015; 28:662-9.

Recebido em 29/Jul/2016

Aprovado em 09/Ago/2016

\section{The authors reply \\ Os autores respondem \\ Los autores responden}

Charles Dalcanale Tesser 1

Thiago Luiz de Campos d'Ávila ${ }^{2}$

1 Universidade Federal de Santa Catarina,

Florianópolis, Brasil.

2 Secretaria Municipal de Saúde de Florianópolis,

Florianópolis, Brasil.

\section{Correspondência}

C.D. Tesser

Centro de Ciências da Saúde,

Universidade Federal de Santa Catarina.

Campus Universitário Trindade, Florianópolis

SC 88040-900, Brasil.

charles.tesser@ufsc.br

\section{Réplica dos autores à carta de Arn Migowski}

Sobre a carta de Arn Migowski a respeito do nosso artigo (Tesser \& d'Ávila 1) e a interpretação das diretrizes nacionais sobre o rastreamento mamográfico ${ }^{2}$, fazemos algumas considerações:

Migowski afirma que "nas novas diretrizes nacionais, essa consistência [efeito positivo leve de redução de mortalidade por câncer] foi valorizada e não as evidências "consensuais" defendidas no ensaio [artigo]". Não identificamos que "evidências consensuais" seriam essas que teríamos defendido.

A crítica de que há uma contradição em nosso artigo é aceitável e a assumimos, subliminarmente, quando discutimos nele os limites da discussão realizada. Usamos o mesmo argumento de Migowski: nos respaldamos nas revisões realizadas para as últimas diretrizes nacionais ${ }^{2}$ com a diferença de que chegamos a conclusões diversas: frente à diversidade, extensão e magnitude dos danos e à pequenez dos benefícios, nosso questionamento foi que parece pouco sustentável e arbitrário considerar o balanço danos $\mathrm{x}$ benefícios favorável, mesmo que com pouca força (nos 50-69 anos) 2.

A carta não ou pouco aborda alguns pontos relevantes: (a) a forte assimetria entre recomendar e não recomendar rastreamentos, que envolve diferenças entre cuidado clínico ao adoecimento e "prevenção aditiva" (conforme conceituada no artigo) relativas à incerteza, tolerância aos danos e atitude em relação à ação; (b) a relevância dos resultados de estudos observacionais nas populações; e (c) a avaliação do balanço entre danos e benefícios (crucial).

O primeiro ponto [(a)] diz respeito à maior força da não-maleficência em relação à beneficência no caso da recomendação de rastreamento mamográfico, devido à menor tolerância à incerteza e aos danos e à maior exigência de garantia de resultados nesse tipo de prevenção. Isso demanda uma mudança de postura e atitude (quase outra subjetividade), exigida nos rastreamentos: do consenso afirmativo e otimista da prevenção redutiva e de um intervencionismo ativista comum no cuidado clínico ao adoecido, deve-se passar na prevenção aditiva a um ceticismo negativista e criterioso recheado de conservadorismo resistente 
à ação ${ }^{1}$. Essa mudança atitudinal necessária exige resistência à realização ou recomendação de realização de procedimentos preventivos aditivos até que se estabeleça com ampla segurança e idoneidade um balanço danos $\mathrm{x}$ benefícios significativamente favorável. O ônus da prova fica com a proposição da ação. Não é necessário provar que o balanço é desfavorável para suspender a recomendação, o que deve ocorrer apenas pela ausência de evidências convergentes de balanço amplamente favorável, por precaução e inércia cética resistente à ação: na dúvida ou na ignorância, o recomendável é não rastrear nem recomendar o rastreamento (o que não muda com a existência de recomendação anterior positiva) 1 .

Migowski desconsidera a ausência ou pobreza de evidências de efeito positivo do rastreamento mamográfico nas populações [(b)], apenas reafirma o pequeno benefício detectado nos ensaios clínicos. Se a segurança sobre o balanço benefícios $\mathrm{x}$ danos somente deve ser proporcionada por revisões de estudos de alta qualidade que ofereçam evidências idôneas sobre os resultados finais envolvendo danos e benefícios dos rastreamentos em ensaios clínicos (estudos experimentais), não se deve por isto desconsiderar os resultados obtidos nas populações (estudos observacionais). Ao contrário, esses últimos são importantes para relativizar e contextualizar os resultados dos primeiros. Sabe-se que os resultados desses são geralmente melhores do que os obtidos na vida real das instituições e profissionais de saúde 3 . Uma vez realizados por décadas programas de rastreamento, deve ser possível detectar seus benefícios em populações reais. No presente caso, os resultados nas populações ganham maior importância, dado que o benefício pequeno em ensaios clínicos torna-se ainda menor devido a essa diferença de resultados entre realidade social e ensaios clínicos. Estudos observacionais (sintetizados no nosso artigo) não detectam esse benefício e alguns dos melhores ensaios clínicos também não 1,2 . Ainda há respeitáveis argumentos de que melhorias na terapêutica parecem ter papel relevante na melhora da mortalidade por câncer de mama 1. Isso deve ser considerado e relativiza os já pequenos benefícios em ensaios clínicos.

Migowski aproxima-se do cerne do problema, que não é a eficácia e sim o balanço danos x benefícios [(c)], ao criticar como flagrantemente frágil o critério da Organização Mundial da Saúde de que dois casos de sobrediagnóstico para cada morte por câncer de mama evitada seriam aceitáveis. Porém, questiona que "arbitrar que dez casos de sobrediagnóstico por uma vida prolongada representa um balanço negativo como parecem sugerir os autores" (5º parágrafo da Carta). Embora nos pareça que essa "sugestão" (associada aos demais danos mencionados no artigo) seja sustentável e defensável, nosso artigo apenas defendeu que a dúvida sobre o balanço está posta incontornavelmente. Migowski afirma que "esses desfechos não são diretamente comparáveis entre si e esse é um limite claro das evidências disponíveis". Mas se o balanço danos x benefícios não cotejar danos e benefícios, o que levará em conta? Afirmar que "não são diretamente comparáveis" significa o quê? O balanço danos $\mathrm{x}$ benefícios deve ser avaliado e isto implica necessariamente, tornar relacionáveis danos com benefícios, e isto sua carta não faz. O que fizemos no artigo foi reunir literatura sobre danos e benefícios. Tal confrontação (com pequeno benefício em ensaios clínicos, duvidosos/inexistentes benefícios em estudos observacionais e grandes e numerosos danos) colocou de forma autoexplicativa, compreensível e sem necessidade de grande fundamentação a constatação de que há pelo menos incerteza e dúvida quando ao citado balanço, e é esta a divergência com as diretrizes nacionais 2 . Nelas essa confrontação (quase igual ao nosso artigo) resultou em conclusões positivas frágeis, pouco argumentadas que apenas afirmam ser o balanço "limítrofe, mas provavelmente favorável" (50-59) e "provavelmente positivo" (60-69) 2 (p. 49).

É necessária uma postura ética e tecnicamente sustentável frente à incerteza, ao limite das evidências, à ignorância, à dúvida: propusemos na discussão inicial do artigo e não foi questionada (sintetizada acima). Sendo ela bem fundamentada, frente à dúvida introduzida no balanço danos $\mathrm{x}$ benefícios, nossa defesa da revisão da recomendação em qualquer idade persiste até que melhores evidências esclareçam e alterem o balanço em sentido claramente favorável.

Talvez estejam presentes implicitamente atitudes e julgamentos inerciais influenciando essa discussão ao contrário do que deveriam: como havia recomendação positiva e um "estado de opinião" douto e leigo quase generalizado sobre a desejabilidade do rastreamento (ao menos nos 50-69 anos) 1, parece haver subliminarmente uma exigência de forte e justificado motivo para o seu cancelamento. Porém, o contrário é necessário, a inversão do ônus da prova: a recomendação deveria cair até que argumentos e evidências fortes e convincentes sustentem um balanço danos $\mathrm{x}$ benefícios consistentemente favorável e não duvidoso (para além de haver algum benefício), o que nem Migowski nem as diretrizes brasileiras e outras instituições produziram. Daí a força e a coerência da defesa da revisão da recomendação positiva.

Finalmente, concordamos que há grande incompreensão e crenças arraigadas sobre o tema, muitas convergências entre nosso artigo e as diretrizes nacionais, e grande necessidade de superar a divulgação massiva de informações equivocadas no país.

1. Tesser CD, d'Ávila TLC. Por que reconsiderar a indicação do rastreamento do câncer de mama? Cad Saúde Pública 2016; 32:e00095914.

2. Instituto Nacional de Câncer José Alencar Gomes da Silva. Diretrizes para a detecção precoce do câncer de mama no Brasil. Rio de Janeiro: Instituto Nacional de Câncer José Alencar Gomes da Silva; 2015.

3. Porzsolt F, Rocha NG, Toledo-Arruda AC, Thomaz TG, Moraes C, Bessa-Guerra TR, et al. Efficacy and effectiveness trials have different goals, use different tools, and generate different messages. Pragmatic and Observational Research 2015; 6:47-54

Recebido em 30/Jul/2016

Aprovado em 09/Ago/2016 